\title{
Differential Impact of Costimulation Blockade on Antigen-Activation of Foxp3-Positive and Negative T Cells in A Model of Type 1 Diabetes
}

\author{
Mark R Rigby ${ }^{1 *}$, Alison Trexler ${ }^{2}$, Danxia Duan², Leslie Kean ${ }^{2}$ and Christian P Larsen ${ }^{2}$
}

${ }^{1}$ Department of Pediatrics, Wells Center in Diabetes Research, Indiana University School of Medicine, Riley Hospital for Children, Indianapolis IN, USA

${ }^{2}$ Department of Surgery, Emory University School of Medicine, Atlanta, USA

\begin{abstract}
Using co stimulatory blockade to prevent activation and activity of beta cell specific effector $\mathrm{T}$ cells is a promising approach for preventing or reversing Type 1 diabetes (T1D). Regulatory T cells are likely critical for the maintenance of long-term tolerance. At present, it is unclear how co-stimulatory blocking agents affect the activity of regulatory $\mathrm{T}$ cells. To better understand the mechanism of costimulation blockade induced tolerance in murine autoimmune diabetes, we evaluated the effect of CD28 and CD154 blockade on both beta cell-specific effector and regulatory T cell responses.

Diabetes transferred by lymphocytes isolated from BDC2.5.NOD mice could be prevented if cells were antigenactivated ex vivo in the presence of CTLA4Ig and anti- CD154. Following antigen-stimulation, both effector (Teffs; FoxP3-) and regulatory (Tregs; FoxP3+) CD4+ T cells upregulated CD25, divided and accumulated. Co-culture with CTLA4Ig and anti-CD154 dampened the quantitative and qualitative Teff response (i.e. cell cycling and CD25 expression), but there was little effect on the Treg response. Adding exogenous IL-2 to such cultures reversed the diabetes protective effect of CD28/CD154 blockade.

These findings suggest that Tregs do not only respond vigorously to antigen, but they rely less on traditional costimulatory signals than Teffs. Understanding the different signaling requirements of Teff and Tregs may facilitate the development and investigation of rational therapeutic interventions in Type1 diabetes that will both quiet diabetogenic effector T cells and enhance regulatory pathways.
\end{abstract}

Keywords: Type 1 diabetes; Regulatory T cells; Immune tolerance; NOD mouse; Costimulation

Abbreviations: CoB: Costimulation Blockade; T1D: Type 1 Diabetes; NOD: Non-Obese Diabetic; MHC: Major Histocompatibility Complex; NRT: Non-Regulatory T cells

\section{Introduction}

In many animal models of allo- and auto-immunity, immune tolerance can be generated by the administration of agents that interfere with $\mathrm{T}$ cell activation. Unfortunately, this has not translated to a successful approach for tolerance induction in humans. In the case of T1D, the concept of using a brief course of immunotherapy to quiet diabetes effector $\mathrm{T}$ cells and engendering immune tolerance is one of the most attractive approaches for disease prevention or reversal. One of the most reliable means to prevent autoimmunity and allograft rejection in rodents is by interfering with the CD28/B7 and CD154/CD40 T cell co-stimulatory pathways [1-4]. Although this approach is successful in inducing tolerance in a vast array of models and conditions, the precise cellular mechanisms involved in subduing effector $\mathrm{T}$ cells and maintaining a nonpathogenic state is unclear. For transition of a quiescent in to a effector $\mathrm{T}$ cell, $\mathrm{T}$ cells must receive signal 1 from $\mathrm{T}$ cell receptor engagement and a second signal via costimulatory or accessory molecule engagement, and without this second signal $\mathrm{T}$ cells are susceptible to deletion or deactivation.

Much of the understanding of the putative roles of co-stimulatory molecules in $\mathrm{T}$ cell activation and tolerance have come from in vitro studies where the $\mathrm{T}$ cell receptor is antibody engaged in the absence or presence of the ligation of select costimulatory or accessory molecules. Likely the most classic example is the observation that $\mathrm{T}$ cells incubated with anti-CD3 and CD28 antibody proliferate vigorously whereas proliferation is minimal with anti-CD3 alone and cells are susceptive to deletion or long-term deactivation (i.e. anergy) [1,5-8]. Although helpful in identifying the roles and pathways of costimulatory molecules, the in vitro findings may not accurately reflect the precise in situ roles of these molecules or the consequence of targeted blockade during antigen presentation by antigen presenting cells. In addition different T cell subtypes (i.e. CD4 vs. CD8 and naïve vs. memory cells) appear to have different requirements and thresholds for signal 1 and signal 2. Therefore it may be difficult and unwise to assume how on intervention may affect one $\mathrm{T}$ cell subtype based on findings from another.

Type 1 diabetes (T1D) is a T cell mediated autoimmune condition and the expression disease appears to be influenced, at least in part, by the balance of beta cell recognizing effector and regulatory $\mathrm{T}$ cells [9-11]. Beta cell-specific autoreactive cells that have escaped central tolerance become activated and are responsible for beta cell destruction. A number of defects in peripheral tolerance are thus also implicated in diabetes pathogenesis, including, deletion and deactivation (i.e. anergy) of autoreactive $\mathrm{T}$ cells and modulation by regulatory $\mathrm{T}$ cells

*Corresponding author: Mark R. Rigby, Department of Pediatrics, Wells Cente in Diabetes Research, Indiana University School of Medicine, Riley Hospital for Children, 705 Riley Hospital Drive, ROC 4270, Indianapolis, IN 46202, USA, Tel: 317-948-7185; Fax: 317-944-7267; E-mail: mrigby@iu.edu

Received December 12, 2012; Accepted February 02, 2013; Published February 07, 2013

Citation: Rigby MR, Trexler A, Duan D, Kean L, Larsen CP (2013) Differentia Impact of Costimulation Blockade on Antigen-Activation of Foxp3-Positive and Negative T Cells in A Model of Type 1 Diabetes. J Diabetes Metab 4: 246 doi:10.4172/2155-6156.1000246

Copyright: ( 2013 Rigby MR, et al. This is an open-access article distributed unde the terms of the Creative Commons Attribution License, which permits unrestricted use, distribution, and reproduction in any medium, provided the original author and source are credited. 
$[9,11]$. Many studies indicate that diabetes is the result of quantitative or qualitative deficiencies in regulatory $\mathrm{T}$ cells, and, conversely, treatment with regulatory $T$ cells can prevent diabetes in animal models of T1D. Tregs may be derived from thymic precursors or evolve from peripheral non-regulatory $\mathrm{T}$ cells, and the circulating pool appears to be maintained through interactions of self peptide: MHC complexes. The suppression of effector responses by Tregs can occur via both cellcell interaction and as a result of soluble mediators, which may depend on the site and type of antigen encounter $[10,12,13]$. Although there is a vast knowledge of the requirements, processes, and pathways for effector cell activation, it is less well understood how Treg function is modified during times of stimulatory antigen-encounter and how Treg numbers and function may be influenced by costimulation blockade therapies.

In our previous studies, we found that we could prevent the adoptive transfer of autoimmune diabetes by highly pathogenic BDC2.5.NOD CD4+ T cells by a short course of in vivo therapy of interfering with CD28 and CD154 (CTLA4-Ig and antiCD154), and that this protection was a result of non-deletional, regulatory tolerance [14]. Our data suggested that this approach induces immune tolerance through dampening antigen specific expansion of effector T cells while permitting regulatory $\mathrm{T}$ cells to become established, providing $\beta$ cells both immediate and long-term protection from diabetogenic $\mathrm{T}$ cells. In order to better understand how the outcome of an immune response can be altered by blocking select $\mathrm{T}$ cell signaling pathways, the goal of this current study was to better define how effector and regulatory $\mathrm{T}$ cells respond to antigen and how these responses can be modified by agents that are capable of tolerance induction.

\section{Materials and Methods}

\section{Mice}

BDC2.5.NOD (BDC.NOD) and nod.scid mice were from on site breeding colonies bred and housed in sterile conditions. BDC.NOD mice were defined via blood phenotype containing B220+ cells and $\mathrm{CD} 3+$ cells that were uniformly $\mathrm{v} \beta 4+$. Studies were conducted in accordance with the Emory University Institutional Animal Care and Use Committee guidelines.

\section{Antibodies}

Fluorochrome-conjugated monoclonal antibodies to CD3, CD4, $v \beta 4, B 220, C D 62 L, C D 25$, flow cytometry were from BD Biosciences/ Pharmingen. Anti-FoxP3 staining kits were from eBiosciences. AntiCD154 (MR1) and CTLA4-Ig used in in vitro cultures were from BioExpress (West Lebanon, NH).

\section{Cell preparation and culture}

BDC.NOD mice were euthanized and spleens and lymph nodes harvested and made into cell suspensions, and washed in RPMI. Cells were adjusted to $1-2$ million cells/ml in RPMI $+10 \%$ FCS and were added to flat bottom cell culture plates (Falcon). When indicated, stimulatory peptide $(10 \mu \mathrm{g} / \mathrm{ml}$; RTRPLWVRME; Emory University Microchemical facility [15], CTLA4-Ig (100ug/ml) and/or anti$\mathrm{CD} 154$ (100ug/ml; MR1)) was added. Cells were incubated at $37^{\circ} \mathrm{C}$ in a humidified incubator containing $5 \% \mathrm{CO}_{2}$ for times indicated in the text. For fluorescent labeling, prior to culture, cells were incubated with $5 \mu \mathrm{mol} / \mathrm{l}$ carboxyfluorescein succinimidyl ester (CFSE, BD Biosceinces) at $37^{\circ} \mathrm{C} \times 10 \mathrm{~min}$, quenched with cold RPMI $+10 \% \mathrm{FCS}$, and washed.

\section{Microscopy}

During cell culture, plates were examined under 100X microscopy using an inverted microscope (Olympus IX70). Still shots were taken with a fitted with a Polaroid DMC2 digital camera and processed with the supplied software.

\section{Flow cytometry}

For qualitative analysis, freshly isolated cells or cultured cells were incubated with directly conjugated antibodies to cell surface molecules for $20 \mathrm{~min}$, and then processed per the manufacturer's instructions for intracellular FoxP3 detection. Cells were then run on an LSRII (BD Biosciences) and data analyzed with FlowJo (TreeStar). Cells cultured in media and stained with directly conjugated antibodies were used to establish gating. For quantitative flow cytometry, prior to or during culture, cells were resuspended, $100 \mu \mathrm{l}$ removed and added to a TruCount Tube, containing $5 \mu \mathrm{l}$ each of surface antibodies and vortexed. After 20 min incubation, $100 \mu$ of stabilizing fixative (BD Biosciences) was added and samples evaluated by flow cytometry. A conversion factor was calculated by dividing the bead number in the tube supplied by the manufacturer by the final volume in the tube (i.e. $225 \mu \mathrm{l}$ ). Following analysis, a ratio of cells to beads was determined. Absolute cell numbers were calculated by multiplying this ration by the conversion factor. For CFSE evaluation cells cultured as indicated were assess by flow cytometry and $\mathrm{CD} 3+, \mathrm{CD} 4+\mathrm{T}$ cells were grouped into either FoxP3+ or FoxP3- subpopulations. CFSE peaks were manually determined and used as a gating strategy for other samples in the same individual experiment.

\section{Adoptive transfer and diabetes detection}

For adoptive transfer cells were prepared as above and cultured in the absence or presence of stimulatory peptide $(10 \mu \mathrm{g} / \mathrm{ml})$, CTLA4Ig $(100 \mu \mathrm{g} / \mathrm{ml})$, MR1 $(100 \mu \mathrm{g} / \mathrm{ml})$ and/or rIL-2 (500 IU/ml). After 4 days in culture, contents of the wells were resuspended, washed twice in RPMI media, and injected via lateral tail vein into NOD.SCID mice. Tail vein blood was analyzed using a Bayer Ascensia Elite Glucometer three times weekly. Diabetes was diagnosed at the first of two consecutive readings $>250 \mathrm{mg} / \mathrm{dL}$.

\section{Cytokine evaluation}

During cell culture, supernatant was removed from wells without disturbing cells. IL-2 content was quantitated using a murine bioplex cytokine assay (BioRad) analyzed on a Luminex 100 IS system according to the manufacturer's instructions.

\section{Statistical Analysis}

Where indicated Student's $t$-test or Fisher's Exact test were used to compare statistical significance. Where multiple comparisons were to be made, ANOVA or exact contingency table analysis was conducted first with post-hoc comparisons of individual groups.

\section{Results}

We have previously demonstrated that in vivo treatment with CTLA4Ig and anti- CD154 to block the CD28/B7 and CD154/CD40 pathways can prevent diabetes in nod.scid adoptive transfer recipients of diabetogenic BDC cells. To determine if costimulation blockade could also influence the pathogenicity of ex vivo antigen activated $\mathrm{T}$ cells, BDC cells were incubated with stimulatory peptide in the absence and presence of individual or combined costimulation blockade and then transferred into NOD.SCID mice (Figure 1). Recipients of cells incubated with or without stimulatory peptide reliably developed diabetes. Diabetes development was minimally impacted when peptide stimulation occurred with anti-CD154 treatment alone and somewhat 


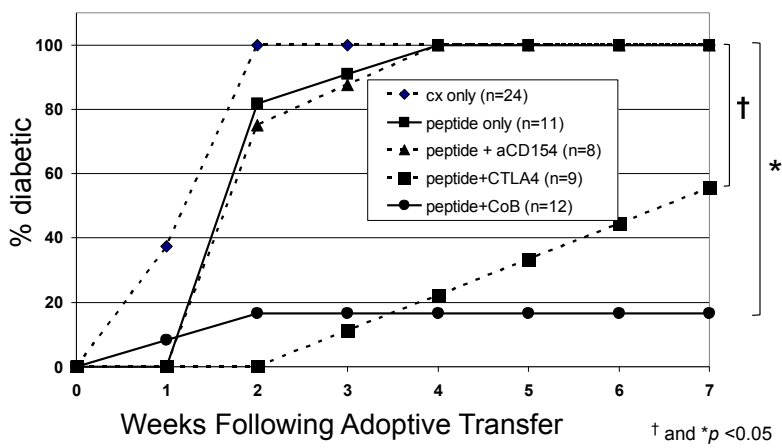

Figure 1: Adoptive transfer of in vitro, peptide-activated BDC cells can be prevented by co-culture with CTLA4Ig and anti-CD154. Lymphocytes were harvested from BDC2.5.NOD mice and cultured for 4 days in the absence or presence of stimulatory peptide with or without CTLA4lg and/or anti-CD154. $2.5 \times 106$ cells were then transferred intravenously to nod.scid recipients that were followed for diabetes. $\mathrm{Cx}$ only = cells cultured alone without peptide, CTLA4Ig or anti-CD154. CoB $=$ the combination of costimulation blockade agents, CTLA4Ig and anti-CD154. $p<0.001$ when comparing diabetes rates at 7 weeks in peptide only, peptide+CTAL4Ig, and peptide $=$ CoB groups using exact contingency table analysis. In post-hoc Fisher exact test analysis, $p<$ $0.001\left(^{*}\right)$ and $p=0.026(\dagger)$ comparing diabetes rates in peptide only group to peptide $+c \mathrm{CoB}$ or peptide $+\mathrm{CTAL} 4 \mathrm{Ig}$ groups respectively.

slowed by CTLA4Ig alone. Yet the recipients of cells antigen-activated in the presence of both CTLA4Ig and anti-CD154 (combine costimulation blockade $(\mathrm{cCoB}))$ were regularly protected from diabetes. This data suggests that the previously observed in vivo tolerogenic effects of combined CD28/CD154 blockade can be recapitulated during antigenspecific encounter in vitro.

To investigate the effect of costimulation blockade on the activation of $\mathrm{T}$ cells by specific antigen, BDC cells were incubated for up to 4 days in the absence or presence of stimulatory peptide, with or without individual or combined costimulation blockade and evaluated. T cells incubated with peptide with our without combined blockade underwent blast transformation and clumping consistent with activation (Figure 2). When CD4+ T cell accumulation was assessed by quantitative flow cytometry, in all culture conditions that contained stimulatory peptide, there was substantial expansion of total CD4+ T cells (Figure 3). In the presence of costimulation blockade, total $\mathrm{T}$ cell numbers were reduced to $50-60 \%$ of maximum numbers seen with peptide-only stimulation. Total T cell expansion was not noticeably affected by anti-CD154 alone and intermediately reduced by CTLA4Ig alone (data not shown). These data indicate that, even in the presence of agents that interfere with pathways considered integral for $\mathrm{T}$ cell activation, $\mathrm{T}$ cells may still go through some of the early processes associated with activation (i.e., blast transformation and proliferation) following specific antigen encounter.

Altering the balance of effector and regulatory $\mathrm{T}$ cells can influence diabetes susceptibility or protection. In BCD2.5.NOD mice approximately $10-15 \%$ of CD4+ T cells are FoxP3+ regulatory $\mathrm{T}$ cells (Tregs). We examined how regulatory and non-regulatory $\mathrm{T}$ cell subpopulations were affected by stimulatory antigen and costimulation antagonism. Following antigen stimulation, in terms of absolute numbers, both non-regulatory T cells (NRTs) and Tregs expanded substantially from baseline numbers in all conditions containing stimulatory peptide (Figure 3 ). Yet there was a relatively higher accumulation of NRTs over Tregs with peptide stimulation demonstrated as a lower percentage FoxP3+ cells compared to input cells. In the presence of combined costimulation blockade (Figure 3), maximal NRT numbers were significantly and substantially reduced, whereas there was actually an increase in Treg numbers, resulting in a relatively higher percentage of Tregs. This data suggests that in response to antigen-specific stimulation, CD28/CD154 blockade relatively specifically retards the expansion and development of effector cells with a minimal quantitative effect on Tregs expansion. The net effect of combined blockade during antigen stimulation is a higher Treg to Teff ratio than with antigen stimulation alone.

We then assessed how costimulation blockade affected the initial proliferative responses of antigen activated $\mathrm{T}$ cell subpopulations by CFSE content (Figure 4). One day following antigen exposure (Figure $4 \mathrm{a}$ ), cell cycling had not begun; yet by day 3 (Figures $4 \mathrm{a}$ and $4 \mathrm{~b}$ ), the majority of NRTs and Tregs had gone through several divisions. At day 3, with peptide stimulation only, more NRTs had gone through a greater number of divisions than Tregs. For example, as shown in histograms (4a) and graphically (4b), although most NRTs and Tregs have had at least 1 division, approximately $40 \%$ of NRTs have gone through at least 4 divisions, compared to $\sim 15 \%$ of Tregs. Costimulation blockade appeared to preferentially dampen the intermediate cycling of NRTs. At day 3 almost all (>90\%) NRTs stimulated in the presence of $\mathrm{CoB}$ have gone through at least 1 division, yet a minority (i.e. 20\%) of cells has gone through 4 or more divisions (Figures $4 \mathrm{a}$ and $4 \mathrm{~b}$ ). In sharp contrast, the division kinetics of peptide stimulated Tregs is not noticeably altered by costimulation blockade (Figures $4 \mathrm{a}$ and $4 \mathrm{~b}$ ).

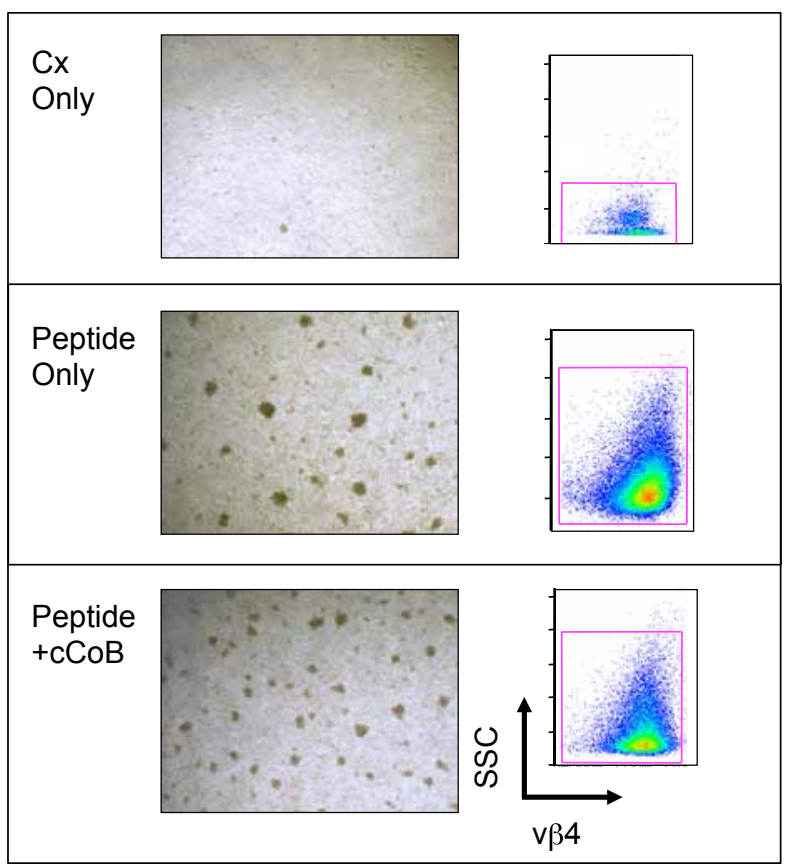

Figure 2: Peptide-stimulated BDC cells are activated in the presence of CTLA4Ig and anti-CD154. BDC cells were cultured for 3 days in the absence (culture (cx) only) or presence of stimulatory peptide without (Peptide Only) or with CTLA4Ig and anti-CD154 (combined costimulation blockade (cCoB)). Photomicrographs of cultured cells were taken (left panel). In addition cells were stained with $C D 3, C D 4$ and $v \beta 4$ - florochrome-conjugated antibodies. $\mathrm{CD} 3+, \mathrm{CD} 4+$ and $\mathrm{v} \beta 4+$ cells were identified via flow cytometry (where $\mathrm{v} \beta 4+$ cells were regularly $>90 \%$ of the CD3+, CD4+ population) and the side-scatted (SSC) versus $\vee \beta 4$ cells are shown (right panels). Peptide stimulate cells even in the presence of costimulation blockade go though some of the processes of activation, including blastogenesis and clumping. Shown are representative of more than 6 experiments. 

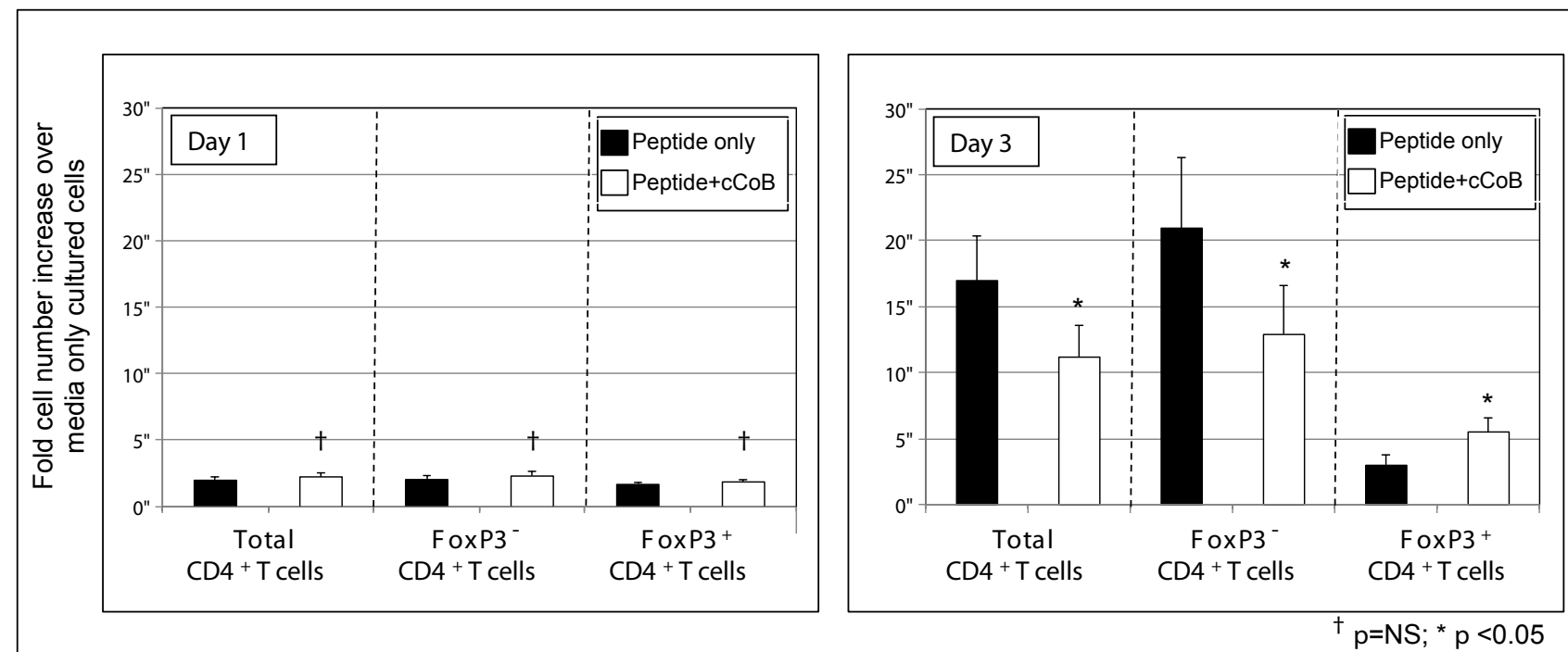

Figure 3: Costimulation blockade preferentially targets expansion of peptide stimulated non-regulatory $\mathrm{T}$ cells over regulatory $\mathrm{T}$ cells. BDC were activated in the absence (media only) or presence of stimulatory peptide without (peptide only) or with combined costimulation blockade (peptide+cCoB) for 1 and 3 days. Cells were evaluated by quantitative flow cytometry where absolute cell numbers were assessed using TruCount beads. CD3+ CD4+ T cells were identified (Total CD4+ T cells), or subpopulated based on FoxP3- and FoxP3+ CD4+ T cells. Shown are data from compiled from 3 independent experiments where T cell numbers were standardized to cells cultured without peptide and fold differences in the indicated subpopulations were calculated, and means and standard deviations (error bars) of these fold differences are displayed. Pairwise comparisons of the indicated T cell subpopulations were analyzed by Student's t-Test and $\mathrm{p}$ values are shown.

The results thus far suggest a selective, quantitative dampening effect of costimulation blockade on the generation of Teffs over Tregs. We next asked if there were qualitative repercussions of costimulation blockade on developing effector and regulatory $\mathrm{T}$ cells. CD25 is the alpha component of the IL-2 receptor complex, and is rapidly upregulated during $\mathrm{T}$ cell activation and integral to $\mathrm{T}$ cell expansion and differentiation. Wells et al. has demonstrated that interfering with CD28 signaling (using CTLA4Ig) during polyclonal stimulation of T cells slows cell division and reduces CD25 expression [16]. CD62L is an adhesion molecule expressed primarily by naïve $\mathrm{T}$ cells, assists with lymph node trafficking and is shed following activation. We assessed CD25 and CD62L expression on NRT and regulatory cells before (Figure $5 \mathrm{a}$ ) and at different division stages following peptide activation in the absence and presence of costimulation blockade (Figures $5 \mathrm{~b}$ and 5c). At 24 hours, before any division has occurred, CD25 is highly upregulated on virtually all NRTs and Tregs cultured with peptide, irrespective of costimulation blockade (Figure 5b). After 3 days, CD25 expression remains highly expressed on NRTs and Tregs stimulated with peptide only, regardless of division stage (i.e. CFSE content). Conversely CD25 expression on RTs substantially reduced on antigenactivated NRTs cultured with costimulation blockade. When CD25 expression is plotted according to divisional stage at day 0,1 , and day 3 (Figure 6) it's clear to see that CD25 increases on Tregs and remained highly expressed no matter the day nor divisional stage following antigen encounter, irrespective of the addition of CTLA4Ig and antiCD154 (Figure 6 solid lines). In sharp contrast, on NTRs activated in the presence of combined costimulation blockade, CD25 expression peaks but then is sequentially diminished over time with progressive cell divisions (Figure 6 dashed lines).

In comparison, there was no apparent regular selective impact of costimulation blockade on CD62L expression on NRTs or Tregs during activation (Figure 5c).

IL-2, produced either in an autocrine or paracrine manner, is integral in the activation and expansion of effector T cells. TCR and costimulatory signals promote expression of the IL-2R and IL-2 transcription [17]. To investigate the effect of costimulation blockade on IL-2 production in our model, we assessed IL-2 levels in culture supernatants of cells activated in the presence and absence of costimulation blockade (Figure 7a). As expected, with peptide stimulation alone IL-2 was found in abundance in supernatants 2 days following stimulation. By day 3, IL-2 levels were radically diminished in these cultures which likely reflect IL2 consumption in the expanding cells and/or the negative feedback of IL2 production to prevent uncontrolled T cell expansion. Little IL-2 was detected on any day in non-stimulated cultures or those stimulated in the presence of combined blockade despite significant turnover of both NTR and Tregs.

To ascertain if reconstituting IL-2 could reverse protective effects of combined blockade on ex vivo stimulated cells, BDC cells were peptidestimulated in the presence of combined blockade and exogenous IL-2 (Figure 7b). Adoptive transfer recipients of these cells uniformly developed diabetes at a rate and tempo similar to recipients of cells stimulated with peptide alone.

\section{Discussion}

In this report we find that we can prevent the diabetogenicity of $e x$ vivo antigen activated BDC T cells by concomitant blockade of the CD28 and CD154 pathways. Similar to ours in vivo model, ex vivo blocking either pathway alone is less effective and reliable than combined blockade. It is known from a variety of allo- and autoimmune models that these two immunosuppressive agents can cooperate in a unique manner in to suppress effector $\mathrm{T}$ cell response and produce a state of long-lasting immune protection. Despite these multiple observations, relatively little is known of the immunologic processes responsible for this effect. What this work provides is important mechanistic insight as to how antigen activated effector and regulatory $\mathrm{T}$ cells appear to be differentially affected by CD28/CD154 blockade. CD28 was one of the first costimulatory molecules described, as cross-linking with TCR 
Citation: Rigby MR, Trexler A, Duan D, Kean L, Larsen CP (2013) Differential Impact of Costimulation Blockade on Antigen-Activation of Foxp3Positive and Negative T Cells in A Model of Type 1 Diabetes. J Diabetes Metab 4: 246. doi:10.4172/2155-6156.1000246

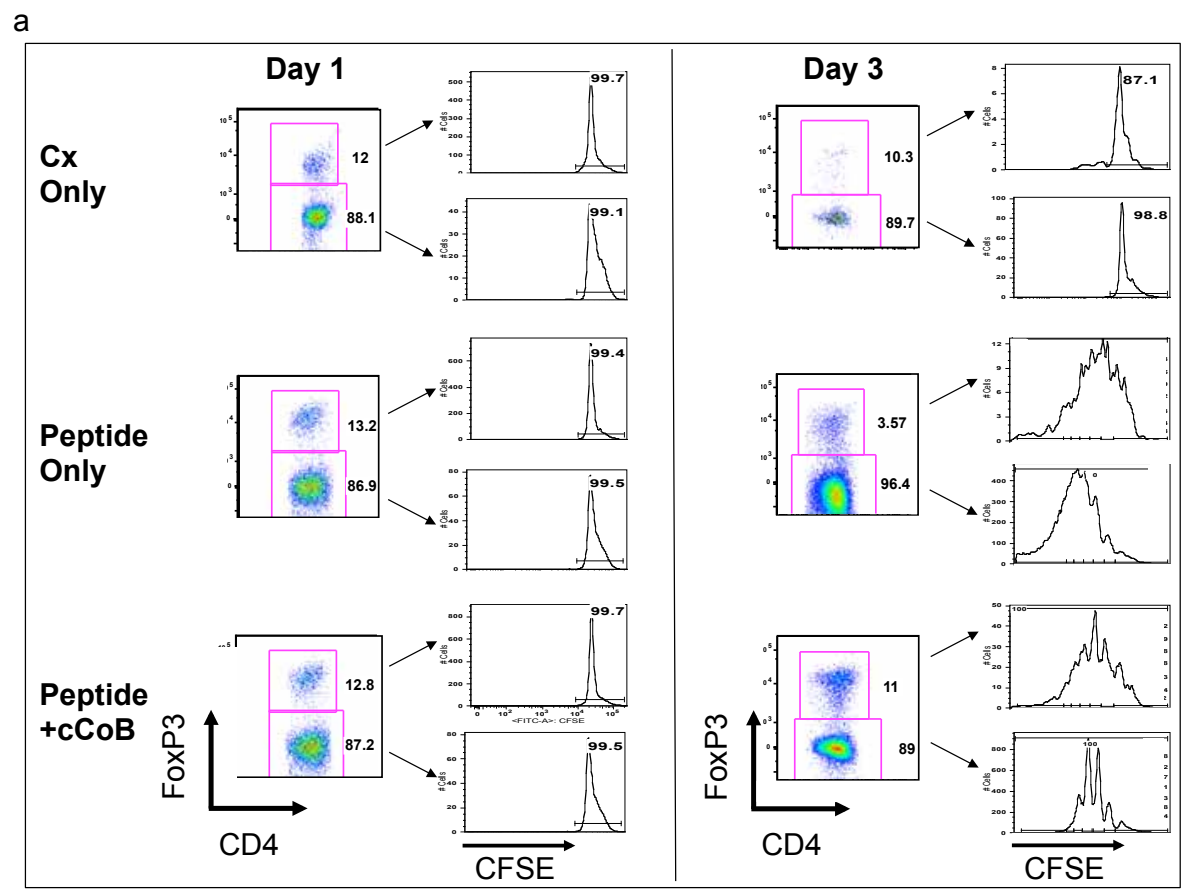

b

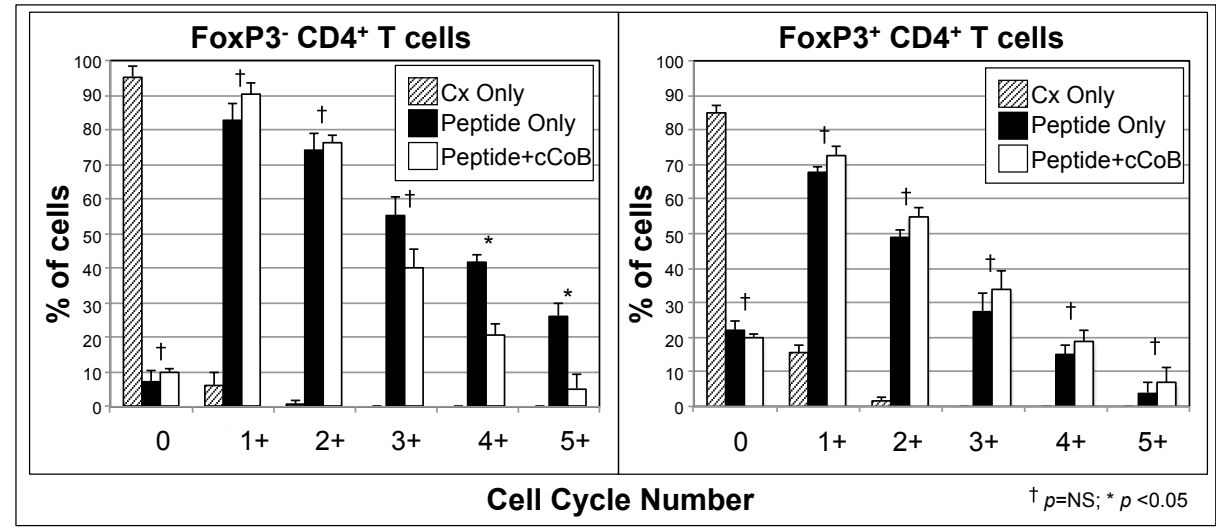

Figure 4: Impact of costimulation blockade on division of non-regulatory and regulatory T cells. BDC cells were labeled with CFSE and cultured with or without stimulatory peptide and costimulation blockade. (A) At 1 and 3 days cells were analyzed for CFSE content to determine cell divisions. At the indicated days cells cultured as indicated were stained with anti-CD3, CD4, and FoxP3 and assessed by flow cytometry. CD3+, CD4+ cells were separated based on FoxP3 positivity and CFSE content was assessed in the indicated $T$ cell subpopulations. Shown are representative histograms. (B) Cell cycling was determined by quantitating the percentage of cells that had processes though the indicated generations (i.e. $0=$ no divisions, $1+=$ at least 1 cell division, etc). Means and standard deviations (error bars) of percentages of cell subpopulations from 3 experiments are shown. ANOVA analysis was conducted on the 3 treatment groups (culture only, peptide only, peptide+cCoB) in each generation grouping. Post hoc Student's t-Test was used to compare percentage of cells in the peptide only and peptide $+c C o B$ groups and $p$ values of $>0.05$ (NS) or $<0.05$ are indicated using $\dagger$ and ${ }^{*}$, respectively.

induced $\mathrm{T}$ cell proliferation [7,11,18-20]. Due to the findings from such in vitro studies, CD28 signaling is often considered "requisite' for generating an effective $\mathrm{T}$ cell response. Yet from some in vivo studies the absolute role CD28 in nascent immune responses is less clear. For example CD28 knockout NOD mice rapidly develop diabetes at an early age $[9,21]$. Although this high incidence of diabetes is linked to the absence of regulatory $\mathrm{T}$ cells, the fact that $\mathrm{T}$ cells from these mice are quite capable of orchestrating the destruction of beta cells indicate that CD28 is not always required for the generation of effector T cells. On the other hand, CTLA4Ig and antagonistic antibodies to CD80 and CD86, can impair allo and auto-immune responses in non- knockout models [3,22-26]. Currently CTLA4Ig (Abatacept) which is FDA approved for rheumatoid arthritis, has shown success in juvenile idiopathic arthritis, and has shown promise in newly diagnosed T1D $[1,4,27]$. A sequence variant of CTLA4Ig, LEA29Y (Belatacept) is in clinical trials for transplantation $[4,27,28]$.

CD154 also appears integral to the $\mathrm{T}$ cell response and is induced on T cells following TCR engagement and binds to CD40 constitutively expresses on APCs. Anti-CD154 alone can delay allograft rejection and autoimmunity and NOD CD154-knockout mice are nearly fully protected from diabetes [29-32]. Due to its small cytoplasmic domain, 
Citation: Rigby MR, Trexler A, Duan D, Kean L, Larsen CP (2013) Differential Impact of Costimulation Blockade on Antigen-Activation of Foxp3Positive and Negative T Cells in A Model of Type 1 Diabetes. J Diabetes Metab 4: 246. doi:10.4172/2155-6156.1000246

it is unclear to what extent CD154 directly participates in T cell activation, but blocking may interfere with cognate $\mathrm{B}$ cell and dendritic cells $[1,33,34]$. Clinical evaluation of blocking the CD154 pathway was derailed because anti-CD154 can lead to thromboembolism, likely secondary platelet activation [1,35]. Identifying alternative means to interfere with the CD154:CD40 pathway (i.e. targeting CD40 and
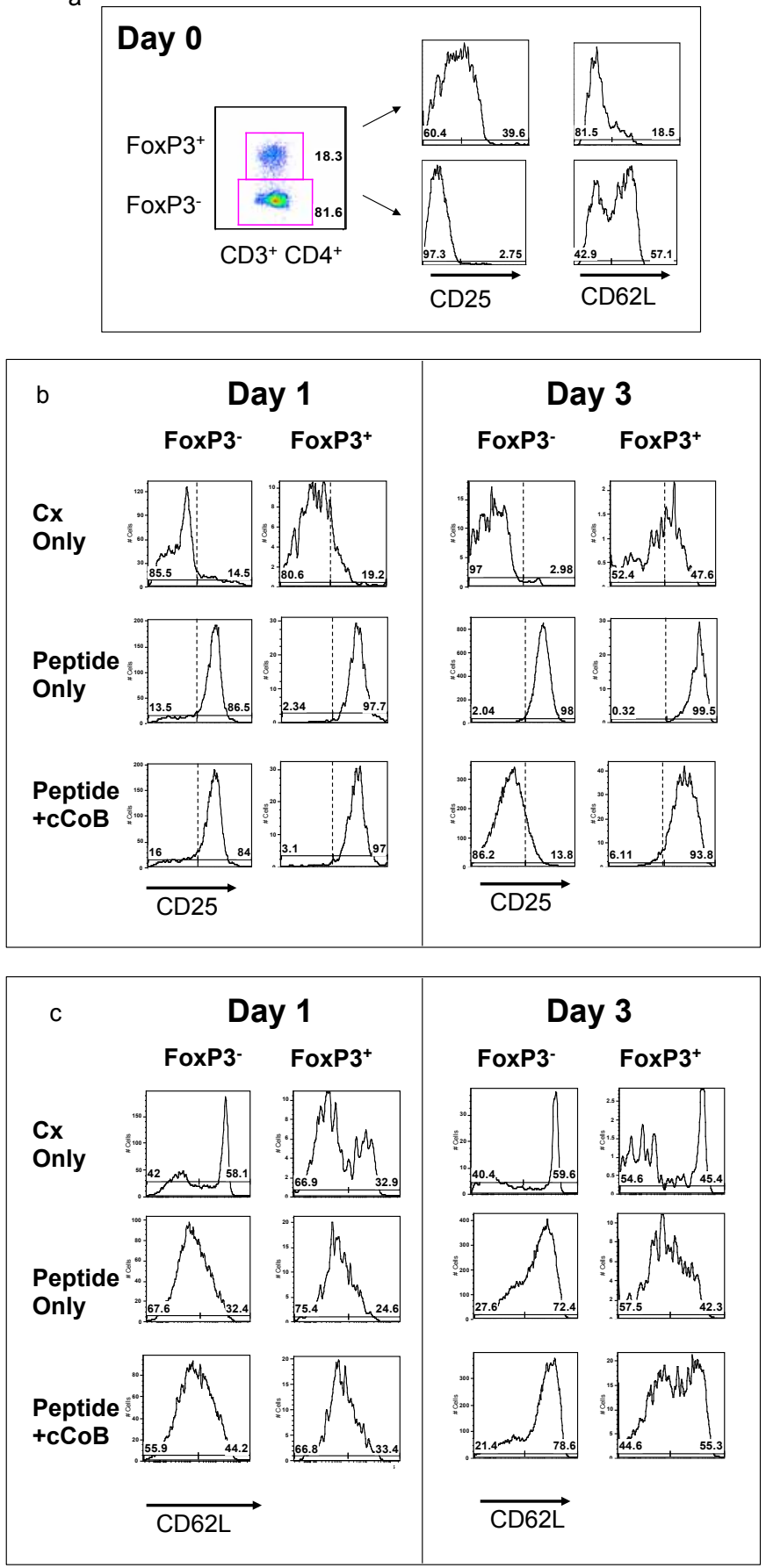

Figure 5: CD25 is reduced on non-regulatory T cells yet spared on regulatory $T$ cells in the presence of costimulation blockade. CD25 and CD62L was assessed on CD3+CD4+ FoxP3- and FoxP3+ cells from (A) freshly isolated cells $(B)$ following 1 day of culture or (C) 3 days of culture under the conditions indications. Shown are results from one of 4 independent experiments.

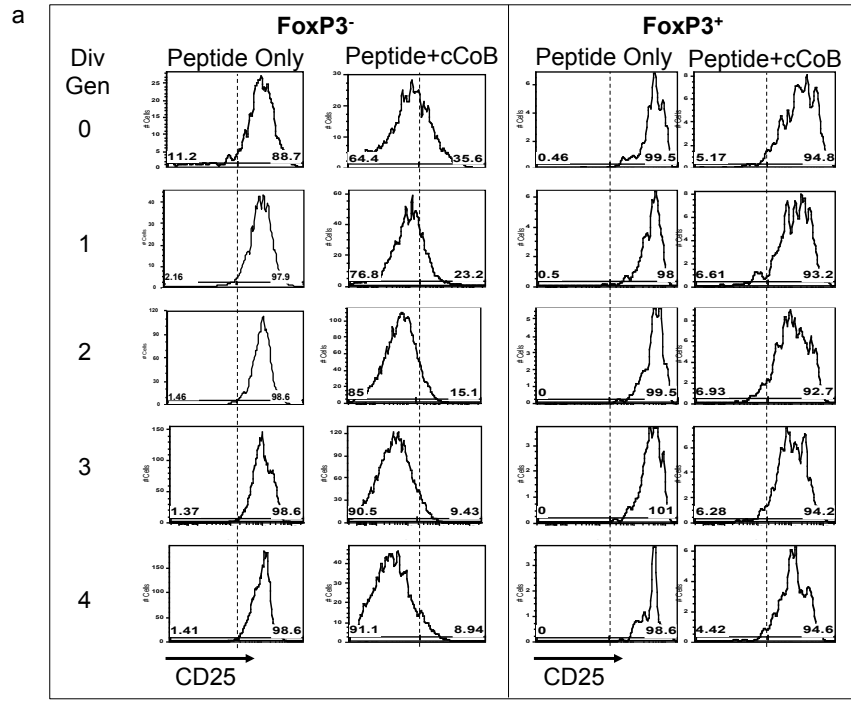

b

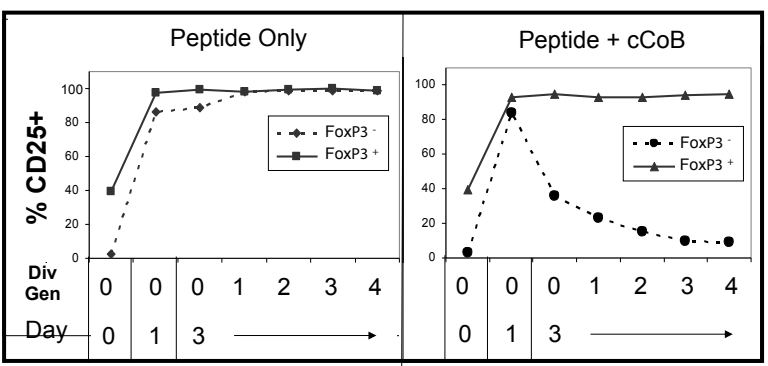

Figure 6: Kinetics of CD25 expression of non-regulatory and regulatory $T$ cells of cells activated in the absence and presence of costimulation blockade. CD25 was evaluated on sequential divisions of FoxP- and FoxP3+CD3+CD4+ $T$ cells stimulated in the absence or presence of costimulation blockade and histograms are shown (A). CD25 expression was assessed of on FoxP3 and FoxP3+ subpopulations of freshly isolated (day 0 ) and following 1 day of culture and 3 days of culture according to divisional generation (Div Gen) assessed by CFSE under the conditions shown. Shown are results from one of 3 independent experiments.

alternative CD154 blockers) are being actively investigated [1,27] Further study of this pathway is therefore important and justified.

Despite many studies utilizing these approaches to quiesce immune responses, relatively little is known about the effects of CD28 and CD154 blockade affects in normal $\mathrm{T}$ cell responses and can lead to immune tolerance. Wells et al. [36] demonstrated that although T cells in bulk splenocytes do proliferate with anti-TCR monoclonal antibody, cell division can be accelerated with agonistic anti-CD28 and dampened (but not eliminated) using CTLA4Ig (16). In these studies, CD25 was upregulated most abundantly on anti TCR + anti CD28 stimulated $\mathrm{T}$ cells and lowest on in those cultured with anti-TCR and CTLA4Ig and supplementation with exogenous IL-2 in the CTLAIg containing cultures restored CD25 expression and proliferation. In subsequent studies, they concluded that "anergy" (as defined by resistance to subsequent stimulation) may take place following TCR and CD28 stimulation if cells fail to proliferate or when TCR receptor ligation occurs in the absence of CD28 signaling irrespective of proliferation. Although such studies have greatly assisted in evaluating the role of costimulation in T cell activation and expansion, it still remains unclear how interfering with select costimulatory pathways affects select $\mathrm{T}$ cell 

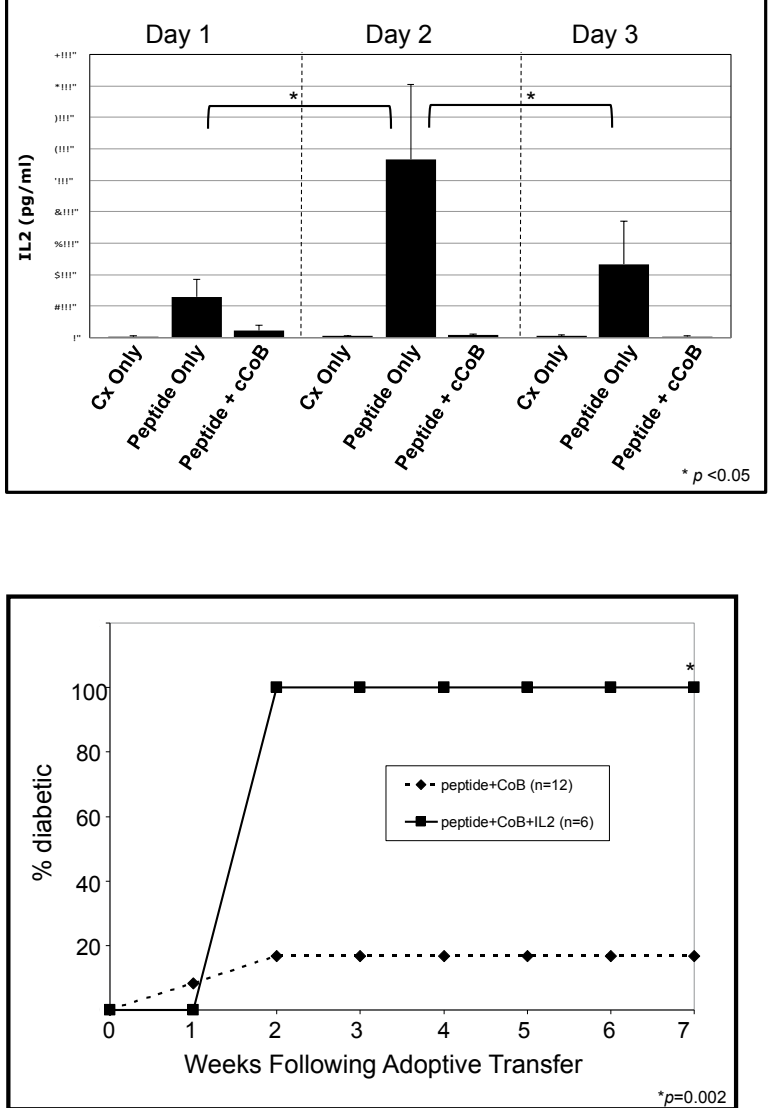

Figure 7: IL-2 is suppressed in BDC cultures stimulated in the presence of costimulation blockade and reconstitution re-engenders diabetogenicity. $(A)$ $\mathrm{BDC}$ cells were cultured in the absence or presence of stimulatory peptide with or without CTL4Ig and anti-CD154. At 1, 2, and 3 days a sample of supernatant was removed and quantitated for IL-2. Shown are the mean IL2 Levels and standard deviation from 3 independent experiments. ANOVA then post hoc Student's t-Test ( $p$ values displayed) was conducted to compare IL 2 levels on day 1 vs. 2 and day 2 vs. 3 on the peptide only cultures. (B) Exogenous IL-2 was added to BDC cells cultured in the presence of stimulatory peptide and CTLA4Ig and anti-CD154, then 2.5 x 106 cells were intravenously transferred to nod.scid mice that were serially assessed for diabetes. For comparison shown are also the results of recipients of cells stimulated in the presence of combined costimulation blockade. Fishers Exact test was used to compare diabetes incidence at 7 weeks.

subtypes (i.e. CD4 vs. CD8, Tregs vs. Teffs) during an in situ response and how such approaches modify a pathogenic immune response into a toleragenic response.

In this report we believe we are the first to report that combined CD28/CD154 blockade suppress the pathogenicity of ex vivo antigenactivated $\mathrm{T}$ cells in an animal model of autoimmune diabetes, and focus on how this intervention effects Teff and Treg responses.

We compared both qualitatively and quantitatively how regulatory $\mathrm{T}$ cell and (pre) effector T cell subpopulations (that we refer to as "nonregulatory" T (NRT) cells) respond to the same antigenic challenge. Many consider Tregs to be minimally responsive to antigen and are relatively passive during the early phases of an immune response $[37,38]$. We find that Tregs respond to antigen encounter in many ways like NRTs. In response to antigen-encounter, Tregs promptly upregulate CD25 (even before division) and divide and accumulate over time. Comparatively, slightly more NRTs appear to cycle than Tregs, and more NRTs divided to greater extent than Tregs - which may be responsible for the greater expansion of NTR. Yet additional factors such as greater activation induced death in the Treg population may also impact the final cell numbers. Therefore although there may be some "absolute" differences in the way Teffs and Tregs respond to antigen (i.e. production of IL-2), there appear to be some responses that are nearly indistinguishable (i.e. rapid regulation of CD25) and some that differ in degree (i.e. proliferation, cell accumulation, activation induced death). Despite similarities in antigen responsiveness, the degree of the robustness of the proliferative response to a specific antigen appears to be greater in NRTs than Tregs. This inherent stochastic advantage of the inherent Teff response to "stimulatory" antigen may favor the activation and accumulation of Teffs over Tregs, and therefore pathogenesis.

Although the default immune response to certain "stimulatory" antigens may be Teff "predominant" (and thus pathogenic), our findings suggest that effector $\mathrm{T}$ cells and Treg have different costimulatory requirements and therefore the outcome of antigen encounter may be modifiable by agents that interfere with accessory $\mathrm{T}$ cell signals.

Specifically our findings suggest that the activation and generation of Tregs is significantly less dependent on CD28 and CD154 than Teffs. In our study, we confirm and extend previous results that CTLA4Ig dampens the proliferative response of TCRengaged T cells. We demonstrate that CTLA4Ig alone or in combination with antiCD154, suppresses the division and accumulation Teffs, and impairs the pathogenicity of these cells. In contrast, costimulation blockade has little impact on the division, expansion, and accumulation of antigen-activated Tregs. Therefore CD28 antagonism, and to a greater extent combined CD28/CD154 blockade, appears to directly influence the net balance of the number Teff and Tregs following activating antigen exposure. It is well known that CD154 blockade, alone or in combination, can prevent or delay allo- or autoimmunity in animal models. Interestingly we find that independent blockade of CD154 has neither substantial impact on the division nor accumulation of NRTs or Tregs following antigen stimulation. CD154 blockade alone nominally reduces IL-2 production and augments CD28 blockade depression of IL-2 production and CD25 expression. Although CD154 has been defined as one of the significant $\mathrm{T}$ cell costimulatory molecules, its blocking has little effect on many of the outcomes usually associated with activation (i.e. $\mathrm{T}$ cell proliferation). This may suggest that during normal immune responses CD154 is involved with aspects of T cell activation other than proliferation (i.e. cytokine production) or effect other facets of the immune response, for example CD40-medaited APC maturation or activation. The fact that immuno-modulatory agents which may have powerful in vivo effects may minimally impact select in vitro readout of $\mathrm{T}$ cell activation (i.e. proliferative), may suggest that these assays may not serve as effective screening methods to identify clinically important immunosuppressive or tolerance induction agents.

In addition to affecting the quantitative balance of Teffs and Treg following antigen encounter, costimulation blockade appears to selectively suppress functional aspects of effector T cells. CD25 is the alpha component of the high affinity IL-2- receptor, and its expression is increased on T cells following TCR receptor engagement and allows responsiveness to IL-2. Signaling through the IL-2 receptor is considered a prerequisite for the propagation of an effective immune response. Studies from Wells and others showed that CD25 expression 
can be blunted by CD28 blockade on bulk TCR simulated T cells $[16,36]$. We sought to further investigate the effect of CD28 and CD154 blockade on CD25 expression on antigenic-specific CD4+ NRTs and Teffs. 24 hours following antigen encounter, regardless of co-culture with any agent, CD25 is highly upregulated on essentially all NRTs and Tregs, prior to division.

Although initial CD25 expression in all CD4+ T cells appears independent of costimulation blockade, maintenance of CD25 on NRTs requires CD28 and CD154 signaling. In NRTs, CD25 expression is maintained at high levels in each daughter division, yet CD25 levels are depressed in a division-dependent manner in cultures containing CD28 and CD154 blockade. T cell costimulation promotes IL-2 transcription and production, and IL-2 appears critical in the cell division and maintenance of CD25 expression on NRTs. Our studies suggest that IL-2/IL-2R independent division takes place through division 2 or 3 , and then slows in the absence of IL-2. IL-2 appears to assist in maintaining the proliferative response in NRTs following this initial phase. Interestingly, we find little IL- 2 in antigen-stimulated cultures at 4 days, suggesting that after such licensing continued expansion and differentiation of Teffs does not require IL-2.

In sharp contrast to the decrease of CD25 on NRTs stimulated in the presence of costimulation blockade, Tregs maintain high CD25 expression no matter their divisional stage. Therefore at the same time there are fewer absolute numbers of NRTs with lowered ability to expand, expansion and activation level of Tregs is relatively unaffected. This effect of costimulation blockade on surface molecule expression is not a generalized phenomenon, as CD62L on all cells is lost following antigen encounter, and then gradually re-accumulates with time and divisions, irrespective of cell type or the presence of costimulation blockade. As some of the very early steps of effector $\mathrm{T}$ cell generation occur in the presence of CD28/CD154 blockade (i.e. blastogenesis, clustering, CD25 expression and initial cell divisions), costimulation blockade appears to "abort" activation, rather than "preventing" activation or leading to certain cell death. When isolated, these NRTs that have undergone this "abortive activation" still retain the ability to transfer diabetes, and therefore are not rendered "terminally" nonpathogenic (data not shown). We postulate that these "abortively activated" NRTs have reduced immediate pathogenicity and are susceptible to suppression by Tregs.

Although Tregs do not produce IL-2, in some cases paracrine or exogenous IL-2 can drive the expansion of quiescent or TCR stimulated Tregs. Yet our work suggests that Treg proliferation may be less dependent on IL-2 than emerging Teffs or in fact may occur independent of IL-2. In our study, IL-2 is detected at high levels in antigen-only stimulated cultures and minimally in cultures with costimulation blockade. In both of those culture conditions expansion and accumulation of Tregs is similar. One interpretation from this is that in situ, peripheral Treg activation and expansion following antigen encounter is critically dependent on CD28, CD154, nor IL-2. Although CD28 is critical in the thymic development of Tregs [10,39-41], this same pathway may not be integral in the function of established Tregs. This may seem to contrast the common notion that IL-2 and perhaps CD28 is needed for Treg expansion and function. Some of this belief may stem from the observation that regulatory $\mathrm{T}$ cell, in humans and rodent, appear to need CD28 signals for homeostasis, as blocking CD28 in non-activated cells or in vivo may reduce Treg numbers. In contrast, our study uses a highly stimulatory antigen, and may suggest that the need for certain costimulatory signals for Tregs is in part impacted by antigen avidity or potency.
In addition, anti-CD28 and exogenous IL-2 has been used to expand Treg ex vivo, but this does not necessarily repudiate our findings. In such approaches it is critical to pre-deplete NTR (i.e. CD3+, CD25 low) to prevent their overgrowth, suggesting that NTR are much more responsive to CD28 and IL-2 than Tregs [13,42,43]. Because CD28 and IL-2 can expand Tregs ex vivo, it does not mean CD28 or IL-2 are critical for in situ, natural, responses. We believe that in our model using a natural admixture of endogenous cells (including $\mathrm{T}$ and B cells, and APCs) and stimulatory antigen more closely resembles an in situ immune response versus the in vitro situation of stimulating sorted CD25+ T cells using non-selective approaches (i.e. anti-CD3). Therefore our study may provide novel insight as to how Teffs and Tregs respond naturally to highly stimulatory antigens in conditions like Type 1 diabetes.

Our findings may be consistent with how some others suggest peripheral Tregs are maintained and function. For example, following specific antigen-MHC encounter (even in the absence of CD28/CD154 signaling), Tregs are able to survive and turnover, and therefore be maintained at a critical mass, even in low IL-2 environments. One putative mechanism by which Tregs suppress immune responses is due to their expression of CD25 (and therefore the high affinity IL-2 receptor), and allowing them to scavenge soluble IL-2 away from effector T cells when antigen- or NTR levels are limiting, and thus slow or prevent their activation, expansion, and pathogenicity $[10,38]$. Yet our study may suggest that during a more robust NTR response (due to higher antigen levels, TCR/MHC avidity or higher NTR precursor frequency) when IL-2 levels are higher, NTR expansion outpaces the suppressive capabilities of Tregs and the effector response predominates. Increasing Treg number and the level of high affinity IL-2 receptor complex on them following antigen-encounter during times of immune quiescence (i.e. non- "danger") may be one means to increase the specificity and robustness of their regulatory response. The natural repertoire of Tregs is highly skewed to self-antigens [10,44,45]. If peripheral Tregs can expand and be maintained in an activated state in the absence of "traditional" costimulation (i.e. CD28, CD154, IL-2), this might be a means to retain a critical mass of T cells which help maintain self-tolerance and prevent autoimmunity. Therefore blocking select signals usually associated with an effective (i.e. pro inflammatory) immune response may selectively subdue effector $\mathrm{T}$ cell generation while allowing the expansion of regulatory process, primarily functional Tregs.

The outcome of an immune response can be influenced by the balance of effector and regulatory $\mathrm{T}$ cells. In the case of autoimmune diabetes, disease can be induced or prevented by altering the balance of diabetogenic or regulatory $\mathrm{T}$ cells $[6,9,21,46]$. We have developed a working model which integrates our current and previous findings regarding how costimulation blockade may affect effector and regulatory $\mathrm{T}$ cell responses to transform a pathogenic response into a protective one. Our data suggest that both pre-effector and regulatory cells can respond vigorously to antigen. With appropriate costimulation, the effector cell response outpaces the Treg response, and, in this model, beta cells are destroyed and diabetes ensues. In the presence of CD28 and CD154 blockade both the number and function of developing effector cells is dampened. By derailing the activation of Teffs, pathogenic effectors are not initially produced and the target tissues (beta cells) are spared. Because of different activation requirements Tregs still expand and function in the presence of blocking agents. The resultant Tregs maintains beta cell protection, and thus tolerance, by suppressing subsequent activation of effector cells. In this scenario costimulation blockade selectively limits the expansion 
Citation: Rigby MR, Trexler A, Duan D, Kean L, Larsen CP (2013) Differential Impact of Costimulation Blockade on Antigen-Activation of Foxp3Positive and Negative T Cells in A Model of Type 1 Diabetes. J Diabetes Metab 4: 246. doi:10.4172/2155-6156.1000246

and activation state of developing Tregs and has little impact on the Treg response to antigen.

It is this relative change in the Teff-Treg balance that is enough to shift the outcome of a pathogenic response to a protective one.

In summary, we believe that this work provides added insight and an important new perspective to how effector and regulatory cells innately respond to specific antigen challenge. Specifically, we hypothesize that because circulating, mature effector and regular $\mathrm{T}$ cell have different absolute or relative (i.e. threshold) costimulation requirements, their expansion, function, and the clinical consequences of antigen encounter can be modified by agents the block select costimulatory pathways, both in vivo and ex vivo. Understanding how to manipulate these responses based on the signaling and the activation requirements of protective and pathogenic $T$ cells could be instrumental in developing strategies which can be translated to control unwanted immune responses, such as Type 1 diabetes.

\section{Acknowledgements}

This project was supported by a Junior Faculty Award from the American Diabetes Association (MRR). Special thanks to Hameeda Bello-Laborn for technical assistance with this project.

\section{References}

1. Weaver TA, Charafeddine AH, Kirk AD (2008) Costimulation blockade: towards clinical application. Front Biosci 13: 2120-2139.

2. Howard LM, Kohm AP, Castaneda CL, Miller SD (2005) Therapeutic blockade of TCR signal transduction and co-stimulation in autoimmune disease. Curr Drug Targets Inflamm Allergy 4: 205-216.

3. Greenwald RJ, Freeman GJ, Sharpe AH (2005) The B7 family revisited. Annu Rev Immunol 23: 515-548.

4. Vincenti $F(2008)$ Costimulation blockade in autoimmunity and transplantation. J Allergy Clin Immunol 121: 299-306.

5. Schwartz RH, Mueller DL, Jenkins MK, Quill H (1989) T-cell clonal anergy. Cold Spring Harb Symp Quant Biol 54: 605-610.

6. Rossini AA, Greiner DL, Mordes JP (1999) Induction of immunologic tolerance for transplantation. Physiol Rev 79: 99-141.

7. Mueller DL, Jenkins MK, Schwartz RH (1989) Clonal expansion versus functional clonal inactivation: a costimulatory signalling pathway determines the outcome of T cell antigen receptor occupancy. Annu Rev Immunol 7: 445480 .

8. Wells AD, Li XC, Strom TB, Turka LA (2001) The role of peripheral T-cell deletion in transplantation tolerance. Philos Trans R Soc Lond B Biol Sci 356: 617-623.

9. Bour-Jordan H, Salomon BL, Thompson HL, Szot GL, Bernhard MR, et al (2004) Costimulation controls diabetes by altering the balance of pathogenic and regulatory T cells. J Clin Invest 114: 979-987.

10. Bour-Jordan H, Bluestone JA (2009) Regulating the regulators: costimulatory signals control the homeostasis and function of regulatory $\mathrm{T}$ cells. Immunol Rev 229: 41-66.

11. Mordes JP, Bortell R, Doukas J, Rigby M, Whalen B, et al. (1996) The BB/ Wor rat and the balance hypothesis of autoimmunity. Diabetes Metab Rev 12: 103-109.

12. Bluestone JA, Tang Q (2005) How do CD4+CD25+ regulatory T cells contro autoimmunity? Curr Opin Immunol 17: 638-642.

13. Brusko TM, Putnam AL, Bluestone JA (2008) Human regulatory T cells: role in autoimmune disease and therapeutic opportunities. Immunol Rev 223: 371 390.

14. Rigby MR, Trexler AM, Pearson TC, Larsen CP (2008) CD28/CD154 blockade prevents autoimmune diabetes by inducing nondeletional tolerance after effector t-cell inhibition and regulatory T-cell expansion. Diabetes 57: 26722683.

15. Judkowski V, Pinilla C, Schroder K, Tucker L, Sarvetnick N, et al. (2001)
Identification of MHC class II-restricted peptide ligands, including a glutamic acid decarboxylase 65 sequence, that stimulate diabetogenic $T$ cells from transgenic BDC2.5 nonobese diabetic mice. J Immunol 166: 908-917.

16. Wells AD, Gudmundsdottir H, Turka LA (1997) Following the fate of individual $T$ cells throughout activation and clonal expansion. Signals from T cell receptor and $\mathrm{CD} 28$ differentially regulate the induction and duration of a proliferative response. J Clin Invest 100: 3173-3183

17. Crispín JC, Tsokos GC (2009) Transcriptional regulation of IL-2 in health and autoimmunity. Autoimmun Rev 8: 190-195.

18. Rossini AA, Mordes JP, Greiner DL (1989) The pathogenesis of autoimmune diabetes mellitus. Curr Opin Immunol 2: 598-603.

19. Rossini AA, Parker DC, Phillips NE, Durie FH, Noelle RJ, et al. (1996) Induction of immunological tolerance to islet allografts. Cell Transplant 5: 49-52.

20. Herold KG, Lenschow DJ, Bluestone JA (1997) CD28/B7 regulation of autoimmune diabetes. Immunol Res 16: 71-84

21. Salomon B, Lenschow DJ, Rhee L, Ashourian N, Singh B, et al. (2000) B7/ CD28 costimulation is essential for the homeostasis of the CD4+CD25+ immunoregulatory $T$ cells that control autoimmune diabetes. Immunity 12: 431 440.

22. Lenschow DJ, Ho SC, Sattar H, Rhee L, Gray G, et al. (1995) Differential effects of anti-B7-1 and anti-B7-2 monoclonal antibody treatment on the development of diabetes in the nonobese diabetic mouse. J Exp Med 181: 1145-1155.

23. Larsen CP, Elwood ET, Alexander DZ, Ritchie SC, Hendrix R, et al. (1996) Long-term acceptance of skin and cardiac allografts after blocking CD40 and CD28 pathways. Nature 381: 434-438.

24. Adams AB, Shirasugi N, Durham MM, Strobert E, Anderson D, et al (2002) Calcineurin inhibitor-free CD28 blockade-based protocol protects allogeneic islets in nonhuman primates. Diabetes 51: 265-270.

25. Boden E, Tang Q, Bour-Jordan H, Bluestone JA (2003) The role of CD28 and CTLA4 in the function and homeostasis of CD4+CD25+ regulatory $\mathrm{T}$ cells Novartis Found Symp 252: 55-63.

26. Tang Q, Smith JA, Szot GL, Zhou P, Alegre ML, et al. (2003) CD28/B7 regulation of anti-CD3-mediated immunosuppression in vivo. J Immunol 170 $1510-1516$.

27. Vincenti F, Kirk AD (2008) What's next in the pipeline. Am J Transplant 8 : 1972-1981.

28. Larsen CP, Pearson TC, Adams AB, Tso P, Shirasugi N, et al. (2005) Rationa development of LEA29Y (belatacept), a high-affinity variant of CTLA4-Ig with potent immunosuppressive properties. Am J Transplant 5: 443-453.

29. Balasa B, Krahl T, Patstone G, Lee J, Tisch R, et al. (1997) CD40 ligand-CD40 interactions are necessary for the initiation of insulitis and diabetes in nonobese diabetic mice. J Immunol 159: 4620-4627.

30. Berney T, Pileggi A, Molano RD, Poggioli R, Zahr E, et al. (2003) The effect of simultaneous CD154 and LFA-1 blockade on the survival of allogeneic isle grafts in nonobese diabetic mice. Transplantation 76: 1669-1674.

31. Molano RD, Pileggi A, Berney T, Poggioli R, Zahr E, et al. (2003) Prolonged islet allograft survival in diabetic NOD mice by targeting CD45RB and CD154. Diabetes 52: 957-964.

32. Rossini AA, Mordes JP, Greiner DL, Stoff JS (2001) Islet cell transplantation tolerance. Transplantation 72: S43-46.

33. Adams AB, Shirasugi N, Jones TR, Durham MM, Strobert EA, et al. (2005) Development of a chimeric anti-CD40 monoclonal antibody that synergizes with LEA29Y to prolong islet allograft survival. J Immunol 174: 542-550.

34. Ford ML, Larsen CP (2009) Translating costimulation blockade to the clinic: lessons learned from three pathways. Immunol Rev 229: 294-306.

35. Kawai T, Andrews D, Colvin RB, Sachs DH, Cosimi AB (2000) Thromboembolic complications after treatment with monoclonal antibody against CD40 ligand. Nat Med 6: 114

36. Wells AD, Walsh MC, Bluestone JA, Turka LA (2001) Signaling through CD28 and CTLA-4 controls two distinct forms of T cell anergy. J Clin Invest 108 895-903.

37. Bluestone JA, Tang Q, Sedwick CE (2008) T regulatory cells in autoimmune diabetes: past challenges, future prospects. J Clin Immunol 28: 677-684 
Citation: Rigby MR, Trexler A, Duan D, Kean L, Larsen CP (2013) Differential Impact of Costimulation Blockade on Antigen-Activation of Foxp3Positive and Negative T Cells in A Model of Type 1 Diabetes. J Diabetes Metab 4: 246. doi:10.4172/2155-6156.1000246

38. Shevach EM (2002) CD4+ CD25+ suppressor T cells: more questions than answers. Nat Rev Immunol 2: 389-400.

39. Liston A, Nutsch KM, Farr AG, Lund JM, Rasmussen JP, et al. (2008) Differentiation of regulatory Foxp3+ T cells in the thymic cortex. Proc Natl Acad Sci U S A 105: 11903-11908.

40. Zheng Y, Rudensky AY (2007) Foxp3 in control of the regulatory T cell lineage. Nat Immunol 8: 457-462.

41. Lenschow DJ, Herold KC, Rhee L, Patel B, Koons A, et al. (1996) CD28/B7 regulation of Th1 and Th2 subsets in the development of autoimmune diabetes. Immunity 5: 285-293.

42. Tang Q, Henriksen KJ, Bi M, Finger EB, Szot G, et al. (2004) In vitro-expanded antigen-specific regulatory $T$ cells suppress autoimmune diabetes. J Exp Med 199: $1455-1465$
43. Putnam AL, Brusko TM, Lee MR, Liu W, Szot GL, et al. (2009) Expansion of human regulatory T-cells from patients with type 1 diabetes. Diabetes 58 652-662.

44. Hsieh CS, Zheng Y, Liang Y, Fontenot JD, Rudensky AY (2006) An intersection between the self-reactive regulatory and nonregulatory $T$ cell receptor repertoires. Nat Immunol 7: 401-410.

45. Hsieh CS, Liang Y, Tyznik AJ, Self SG, Liggitt D, et al. (2004) Recognition of the peripheral self by naturally arising CD25+ CD4+ T cell receptors. Immunity 21: $267-277$.

46. Rossini AA, Handler ES, Greiner DL, Mordes JP (1991) Insulin dependent diabetes mellitus hypothesis of autoimmunity. Autoimmunity 8: 221-235. 